\title{
Protocol on Causal Chain Analysis and Health Economic Modelling of Childhood Anaemia Interventions in Developing Countries - A Health Technology Assessment
}

Mahafroz Khatib¹, Mahalaqua Nazli Khatib², Mahjabeen Ahmed³, Deepak Saxena ${ }^{4}$, Unnikrishnan B. 5 , Shilpa Gaidhane ${ }^{6}$, Abhay M. Gaidhane7, Quazi Syed Zahiruddin ${ }^{8}$

${ }^{1}$ Department of Division of Evidence Synthesis, Centre of Excellence School of Epidemiology and Public Health, Datta Meghe Institute of Medical Sciences (Deemed University), Sawangi Meghe, Wardha, Maharashtra, India.

2Department of Physiology \& Head, Division of Evidence Synthesis, Centre of Excellence School of Epidemiology and Public Health, Datta Meghe Institute of Medical Sciences (Deemed University), Sawangi Meghe, Wardha, Maharashtra, India. ${ }^{3}$ Department of Division of Evidence Synthesis, Centre of Excellence School of Epidemiology and Public Health,

Datta Meghe Institute of Medical Sciences (Deemed University), Sawangi Meghe, Wardha, Maharashtra, India. ${ }^{4}$ Department of Epidemiology, IIPH Gandhinagar, Gujarat ; Adjunct Faculty Datta Meghe Institute of Medical Sciences; Sawangi Meghe, Wardha, Maharashtra State, India; 5 Department of Community Medicine, Kasturba Medical College, Mangalore; Manipal Academy of Higher Education, Manipal, Karnataka, India, ${ }^{6}$ Department of Medicine, Datta Meghe Institute of Medical Sciences, Sawangi Meghe, Wardha, Maharashtra, India. ${ }^{7}$ Department of Community Medicine \& Division of Evidence Synthesis; Centre of Excellence School of Epidemiology and Public Health Datta Meghe Institute of Medical Sciences (Deemed University). Sawangi Meghe, Wardha, Maharashtra, India. ${ }^{8}$ Department of Community Medicine \& Division of Evidence Synthesis; Centre of Excellence School of Epidemiology and Public Health Datta Meghe Institute of Medical Sciences (Deemed University), Sawangi Meghe, Wardha, Maharashtra, India.

\section{ABSTRACT}

\section{BACKGROUND}

As anaemia imposes significant clinical and economic burden in developing countries, health technology assessment (HTA) is intended to explore the most important causal links and the manner in which particular intervention or combination of interventions may lead to fruitful effects for childhood anaemia.

\section{METHODS}

The integrated HTA approach shall include causal chain analysis (CCA), systematic literature review (SLR) and economic modelling. Causal Chain Analysis- Causal chains for anaemia in the children shall be assembled consisting of system characterisation, identification of the activities and the impacts, and their links to the anaemia impacts. Systematic Literature Review- The factors identified by the CCA will define the scope of the SLR and additional factors will be further explored. RCTs and SLRs which evaluated the effect of interventions for childhood anaemia shall be searched and included. An approach proposed by the Cochrane Collaboration and GRADE profiler shall be used. Economic Modelling- Economic model shall estimate the costs/benefits/drawbacks related with childhood anaemia in the developing countries and ensuing influence on the health-care system. The structure will be determined based on available research evidence. The data will be obtained from SLR or other pertinent evidences.

\section{RESULTS}

Results of the Systematic Literature Review, the Causal Chain Analysis, and the Economic Modelling will be combined together to create a consolidated report.

\section{CONCLUSIONS}

HTA shall be useful to policy-makers and health-care personnel in developing completely novel ways of understanding how different intervention effects change in childhood anaemia in developing countries.
Corresponding Author: Dr. Mahalaqua Nazli Khatib, Department of Physiology \& Head Division of Evidence Synthesis, Centre of Excellence School of Epidemiology and Public Health Datta Meghe Institute of Medical Sciences (Deemed University) Sawangi Meghe, Wardha, Maharashtra, India.

E-mail:nazli.786@rediffmail.com

DOI: 10.14260/jemds/2019/845

Financial or Other Competing Interests: None.

How to Cite This Article:

Khatib M, Khatib MN, Ahmed $M$, et al. Protocol on causal chain analysis and health economic modelling of childhood anaemia interventions in developing countries- a health technology assessment. J. Evolution Med. Dent. Sci. 2019;8(51): 3899-3903, DOI:

\section{KEY WORDS}

Childhood Anaemia, Health Technology Assessment, Economic Modelling 


\section{BACKGROUND}

Anaemia is characterized by a decrease in the total amount of haemoglobin or reduction in number of red blood cells, leading to compromised physiological needs of the body.(1) Anaemia in childhood is defined as "a haemoglobin ( $\mathrm{Hb})$ concentration below cut off levels established by the World Health Organization: $<11 \mathrm{~g} / \mathrm{dl}$ in children aged 6-59 months, $<11.5 \mathrm{~g} / \mathrm{dl}$ in children aged 5-11 years and $12 \mathrm{~g} / \mathrm{dl}$ in older children (aged 12-14)".(2)

There are no countries; where anaemia is not at least a mild public health problem.(2) The prevalence of anaemia is higher in low and middle-income countries (LMICs), imposing an economic burden in these countries(3) (Govt. of India 2013a). Irrespective of the aetiology; anaemic states can lead to many impairments associated with growth, cognitive and motor development(4),(5),(6),(7),(8) contributing further to the economic burden on the healthcare system. Anaemia is related with many symptoms such as giddiness, dyspnoea on exertion, and palpitations, all of which affect later affects the quality of life.

The probable cause of childhood anaemia differs in different regions, with iron deficiency anaemia (IDA) being the commonest cause at the global level.(2) It has been estimated that ninety thousand deaths in both sexes and all age groups are due to iron deficiency anaemia alone.(9) However, other nutritional deficiencies (including folate, vitamin $\mathrm{B}_{12}$ and vitamin $\mathrm{A}$ ), inflammation, parasitic infestations, and inherited or acquired disorders that affect haemoglobin synthesis, red blood cell production or survival can lead to anaemia.(2) In developing countries, infectious diseases like malaria, HIV, tuberculosis, and helminthic infestations are other main causes of anaemia.(8),(10),(2) Certain races suffer from inherited forms of anaemia.(11) Sickle cell anaemia is more prevalent in Central African population while $\beta$-thalassaemia is more prevalent in Mediterranean, Middle Eastern and Southeast Asian populations.(12),(11),(8) Iron Deficiency Anaemia (IDA) is common across all age groups, but highest among more vulnerable young children, adolescent girls, pregnant and lactating women.(13) The severity of iron deficiency anaemia is highest during infancy and early childhood.(14) Thus, there exist great disparities across the distribution of disease in different geographies.

Elimination of anaemia is a high priority research area. To be sustainable; prevention and control of anaemia should follow integrated and a long-term approach. According to WHO and UNICEF; preventive strategy for anaemia should be multidisciplinary comprising of food supplementation (In the form of iron-rich diets, iron and folic acid supplementation), food fortification, dietary diversification, prevention and control of malaria and other parasitic infestations, and improving the nutritional status of the community (Baltussen 2004).

Several systematic reviews have evaluated effects of these different interventions addressing anaemia among children and adolescents. There is no consensus in the scientific literature regarding the best regimens for prevention, treatment or control of anaemia. It is important to try and identify the most effective intervention for replication. Understanding the degree to which an intervention 'works' can provide convincing data to policy-makers.
As any intervention aimed at reducing anaemia involves the use of limited resources that could be exploited for other purposes, especially in developing countries, health technology assessment (HTA) is instrumental in establishing the benefits and impact of introducing interventions for addressing childhood anaemia in developing countries. Along with it becomes essential to identify the socio-economic impact due to childhood anaemia and related interventions, so that proper policy interventions bearing greatest benefits for the resource constrained settings of developing countries can be developed. In order to achieve this aim, the HTA through an integrated approach involving causal chain analysis (CCA) will be conducted. This comprises a step-bystep methods that explores causal links between the socioeconomic impacts, their direct causes, activities and economic sectors accountable and, lastly, causes that decide the behaviour of those sectors with respect to childhood anaemia in developing countries. The HTA shall also estimate the costs/benefits/drawbacks associated with interventions for addressing childhood anaemia in the developing countries as well as to evaluate subsequent impact on the health care resources and expenditures through health economic models.

Understanding the effectiveness of an intervention can offer convincing evidence to policy-makers. However; without a supplementary clarification of how an intervention works, it can be challenging to be replicated in other settings, eventually hindering its practicality in making prudent and evidence-informed judgements. As CCA explores the way in which a particular combination of intervention may lead to fruitful outcomes, findings shall be valuable to decisionmakers and practitioners not only in confirming existing theories but also in developing completely novel ways of understanding how interventions bring about a change in the outcomes. This integrated approach based HTA can be of help to policy-makers, paediatricians, physicians, public health experts, programme implementers working in the fields of child health as well as consumers to decide the best possible intervention for preventing and/or treating anaemia in children. Insights from the HTA would support policy makers in proper allocation of scarce resources and interventions to support management of Anaemia in developing countries. Along-with the results shall provide evidence for the policy makers working on introducing interventions which will help reducing Anaemia improving diagnosis, reducing new infections/transmissions and improving linkage to care. This assessment will provide support in communicating the value of the new intervention in developing countries, which can be critical in informing pricing decisions, supporting discussions with different payers as well as reimbursement decision making processes. Development partners can also utilize this overview to notify the requests for research and programme proposals and to support with the valuation of existing and planned programs for improving, preventing and treating childhood anaemias. Clinicians will also find this review valuable in their efforts to campaign for effective preventive and promotional public health strategies.

\section{Review of Literature}

A number of systematic reviews assessing the effects of different interventions for treating childhood anaemia have been conducted. 


\section{HTA Review}

An earlier HTA review by Wilson and colleagues(15) that informed the NICE guidelines (TA1421) assessed the effectiveness including cost-effectiveness of epoetin in cancer-associated anaemia attributable to cancer treatment and concluded that anaemic drugs are effective in improving the haematological response, but that the effect on healthrelated quality of life (HRQoL) is uncertain. A more recent Cochrane review by Tonia and colleagues,(16) concluded that ESAs reduce the need for RBCTs but increase the risk for thromboembolic events and deaths. Another Cochrane review,(17) examined the effect of specific drugs such as ESAs on safety and efficacy outcomes in cancer patients.

\section{Aims and Objectives}

- To explore the most important causal links and the manner in which specific combinations of intervention lead to successful outcomes for childhood anaemia.

- To provide an evidence of the efficacy, costs/benefits/ drawbacks associated with interventions for addressing childhood anaemia as well as to evaluate subsequent impact on the health care system in the developing countries.

\section{METHODS}

The planned integrated HTA approach shall include 3 steps: causal chain analysis (CCA), systematic literature review (SLR) and economic modelling.

\section{Step 1. Causal Chain Analysis}

The methodology to bring together the causal chains for anaemia in children in developing countries shall consist of: (i) system characterisation (site, geography, sociodemographic parameters, culture; etc.); (ii) Detection of the main socio-economic activities in the developing countries; (iii) Detection of key impacts due to anaemia exerted by the economic activities; and (iv) Detection of the main socioeconomic impacts present in the healthcare system in developing countries and their links to the anaemia impacts. The direct causes and the economic activities that generate the anaemia impacts will be identified. Considering the information collected (Socio-economic conditions, impacts of the healthcare system, activities in different sectors and the links between them; etc.), the root causes common to the anaemia will be identified. Based on the parameters CCA shall be performed for two closely associated concerns: childhood anaemia and interventions for childhood anaemia in developing countries.

The causal chain shall be developed through the identification of distal/final outcomes, and then the reviewers shall work backwards to identify or hypothesise the necessary preconditions (intermediate/ proximal/ mediating variables) to reach these distal outcomes. The ultimate aim shall be to create a chain of links between the intervention and the final or endpoint outcome related to childhood anaemia in developing countries.

Different approach, methods or study attribute will be adapted based on the type of causal account being developed. For observation and analysis of process, approach shall include intensive, long-term involvement, collection of rich data, narrative and connecting analysis. With respect to developing and assessing alternative explanations, the preferred approach shall include modus operandi approach, searching for discrepant evidence and negative cases, triangulation and member checks. Various variance approaches comprising of interventions and comparisons shall also be considered.

\section{Step 2. Systematic Review}

Based on the CCA, a review, using a systematic approach, of interventions for the treatment of childhood anaemia will be undertaken. The factors identified by the CCA will define the scope, the inclusion/exclusion of the SLR and the additional factors affecting the childhood anaemia will be further explored through the SLR the results of the SLR will be used further to strengthen the findings of the CCA.

\section{Inclusion Criteria Type of Studies}

Irrespective of the language; RCTs as well as Cochrane/ nonCochrane systematic reviews, which evaluated the effect of various interventions addressing childhood anaemia will be included. Patient-level cost analyses and effectiveness data, cost-effectiveness analyses, cost-utility analyses, cost-benefit analyses and cost-consequence analyses will also be included and assessed.

\section{Type of Population}

We shall include studies of various interventions for preventing, controlling, or treating anaemia amongst children between six months to 10 years. We shall consider studies providing data on interventions targeting anaemia in subgroups by age and included only if they covered the relevant ages. Studies providing information on overlapping age groups will be excluded. No restriction shall be imposed on severity of anaemia, race/ethnicity and type of settings from where the participants were recruited. Studies done on overtly diseased children will be excluded.

\section{Types of Intervention}

Studies which evaluated any one or combination of interventions designed for preventing, controlling, and treating childhood anaemia. Interventions could range from iron/FS/Zn/MMN/vitamins supplementation, iron/FS/Zn/ MMN/vitamins fortification, anti-helminthic treatment (Deworming), treatment/chemoprophylaxis for malaria, $\mathrm{H}$ pylori treatment, WASH (Water, Sanitation, and Hygiene) interventions (UNICEF 2013), and/or combination of these interventions. The comparison groups were either those who did not receive the intervention or those who received a different intervention.

\section{Search Methods for Identification of Reviews}

We will search electronic as well as non-electronic databases.

\section{Following Electronic Databases were searched}

- The Cochrane Library including the Cochrane Database for Systematic Reviews (CDSR),

- Medline via PubMed,

- Excerpta Medica Data Base (EMBASE) database, 
- $\quad$ Cumulative Index to Nursing and Allied Health Literature (CINAHL) database,

- Database of Abstracts of Reviews of Effects (DARE),

- Health Technology Assessment (HTA) database,

- Database of Promoting Health Effectiveness Reviews (DoPHER),

- Web of Science (Thomson Reuters), and

- Websites like Current Controlled Trials, Clinical Trials.gov, FDA website, EMA website.

The searches will be undertaken using search strategies (with free terms and MeSH terms) and the searches will be tailored to individual databases. All searches will be undertaken irrespective of the language of publication. Studies retrieved from searches will be exported into Rayyan web-app for screening. The resource use associated with clinical events will be obtained either from trial data, other published sources. NHS Trusts and experts in the field will be contacted if published sources are not available. Unit cost data will be identified from NHS and PSS reference cost databases, and if not relevant, the unit cost data will be obtained either from published work and/or sponsor submissions to NICE.

\section{Screening Studies}

Studies retrieved from electronic and non-electronic sources (exported in Rayyan web-app) will be screened initially on the basis of title and abstract by two reviewers independently according to the pre-specified inclusion/exclusion criteria. The full-texts of studies found eligible in first phase of screening will then be independently screened for inclusion by two reviewers. Disagreements amongst the primary reviewers will be resolved by a third reviewer.

\section{Data Extraction Strategy}

Two reviewers will independently extract the details about methods and findings of included studies which will be summarised in a tabular format and synthesised narratively. Disagreements amongst the primary reviewers will be resolved by a third reviewer.

\section{Quality Assessment Strategy}

The methodological quality of each included study will be evaluated using appropriate tools depending upon the type of the study. RCTs will be assessed as per Cochrane Risk of Bias tool. Economic evaluations will be critically assessed as per the consensus-developed list of criteria developed by Evers and colleagues.

\section{Step 3. Economic Modelling}

An economic evaluation in the form of economic modelling will be undertaken to estimate the costs/benefits/drawbacks related with interventions for addressing childhood anaemia in developing countries as well as to assess their subsequent impact on the health-care resources and expenditures. Model structure will be decided on the basis of existing evidence and opinions from experts. Analysis of uncertainty will focus on cost-utility. Any uncertainty in the evidence will be explored through sensitivity analysis and, if data permits, through probabilistic sensitivity analysis (PSA). Outputs of PSA will be depicted using plots on the cost-effectiveness plane and costeffectiveness acceptability curves. Results of the Systematic
Literature Review, the Causal Chain Analysis, and the economic modelling will be combined together to create a consolidated report.

\section{Scope}

- The integrated HTA shall address childhood anaemia and interventions addressing childhood anaemia in developing countries. Anaemia and related interventions in other age groups are beyond the scope of the research.

- $\quad$ Also the research is mainly targeted towards developing countries, apart from developing countries other geographies are beyond the scope of the research.

\section{Limitations}

- Though we have tried to undertake a comprehensive search; there is a possibility of missing some literature.

- The findings are limited to developing countries and children and hence the generalisability will be limited.

\section{Implications}

The evidence summarized in this overview can be of help to clinicians, public health experts, policymakers and programme implementers to get a birds eye view of effects and certainty of the evidence supporting these effects exhibited of different interventions targeting childhood anaemia in developing countries with a view to apprise forthcoming programme strategies and help with assessments of programmes for anaemia.
Appendix
Appendix A: Central Search Strategy
Cochrane Database of Systematic Reviews
ID Search
\#1 child*
\#2 children
\#3 infant*
\#4 toddler*
\#5 adolescent*
\#6 girl*
\#7 boy*
\#8 MeSH descriptor: [Child] explode all trees
\#9 MeSH descriptor: [Infant] explode all trees
\#10 MeSH descriptor: [Adolescent] explode all trees
\#11 \#1 OR \#2 OR \#3 OR \#4 OR \#5 OR \#6 OR \#7 OR \#8 OR \#9 OR \#10
\#12 supplement*
\#13 fortification
\#14 fortified
\#15 diversification
\#16 "nutritional intervention*"
\#17 micronutrient*
\#18 anti-helminth
\#19 "helminth control"
\#20 deworming
\#21 WASH
\#22 WIFS
\#23 MeSH descriptor: [Dietary Supplements] explode all trees
\#24 MeSH descriptor: [Food, Fortified] explode all trees
\#25 MeSH descriptor: [Hand Disinfection] explode all trees
\#26 \#12 OR \#13 OR \#14 OR \#15 OR \#16 OR \#17 Or \#18 OR \#19 OR \#20 OR \#21 OR \#22 OR \#23 OR \#24 OR \#25 \#327 \#11 AND \#26 


\section{REFERENCES}

[1] King T. Elsevier's Integrated Pathology. $1^{\text {st }}$ edn. Mosby 2007: p. 263-89.

https://www.sciencedirect.com/science/article/pii/B97 80323043281500176

[2] WHO. The global prevalence of anaemia in 2011. Geneva: World Health Organization, 2015. http://apps.who.int/iris/bitstream/10665/177094/1/9 789241564960_eng.pdf?ua=1

[3] Balarajan Y, Ramakrishnan U, Özaltin E, et al. Anaemia in low-income and middle-income countries. The Lancet 2011;378(9809):2123-35.

[4] Kazadi LA, Ngiyulu RM, Gini-Ehungu JL, et al. Factors associated with growth retardation in children suffering from Sickle Cell Anemia: First Report from Central Africa. Anemia 2017;2017:7916348.

[5] Carter RC, Jacobson JL, Burden MJ, et al. Iron deficiency anemia and cognitive function in infancy. Pediatrics 2010;126(2):e427-34.

[6] Khedr E, Hamed SA, Elbeih E, et al. Iron states and cognitive abilities in young adults: neuropsychological and neurophysiological assessment. Eur Arch Psychiatry Clin Neurosci 2008;258(8):489-96.

[7] Lozoff B. Iron deficiency and child development. Food Nutr Bull 2007;28(Suppl 4):S560-71.

[8] Soliman AT, De Sanctis V, Kalra S. Anemia and growth. Indian J Endocrinol Metab 2014;18(Suppl 1):1-5.

[9] WHO. Global health estimates 2014 summary tables: deaths by cause, age and sex, by WHO region, 20002012, Geneva: World Health Organization, 2014. http://www.who.int/healthinfo/global_burdendisease/e stimates/en/index1.html
[10] Kassebaum NJ, Jasrasaria R, Naghavi M, et al. A systematic analysis of global anemia burden from 1990 to 2010. Blood 2014;123(5):615-24.

[11] Weatherall DJ. The inherited diseases of hemoglobin are an emerging global health burden. Blood 2010;115(22):4331-6.

[12] Williams TN, Weatherall DJ. World distribution, population genetics and health burden of the hemoglobinopathies. Cold Spring Harb Perspect Med 2012;2(9):a011692.

[13] NITI Aayog. National Nutrition Strategy, Government of India. New Delhi, September 2017. http://niti.gov.in/writereaddata/files/document_public ation/Nutrition_Strategy_Booklet.pdf

[14] Joo EY, Kim KY, Kim DH, et al. Iron deficiency anemia in infants and toddlers. Blood Res 2016;51(4):268-73.

[15] Wilson J, Yao GL, Raftery J, et al. A systematic review and economic evaluation of epoetin alpha, epoetin beta and darbepoetin alpha in anaemia associated with cancer, especially that attributable to cancer treatment. Health Technol Assess 2007;11(13):1-202, iii-iv.

[16] Tonia T, Mettler A, Robert N, et al. Erythropoietin or darbepoetin for patients with cancer. Cochrane Database Syst Rev 2012;12:CD003407.

[17] Bohlius J, Schmidlin K, Brillant C, et al. Erythropoietin or darbepoetin for patients with cancer - meta-analysis based on individual patient data. Cochrane Database Syst Rev 2009;(3):CD007303. 